\title{
Meinung
}

Katja Thiele* und Britta Klagge

\section{Öffentliche Bibliotheken als dritte Orte und Bildungsgerechtigkeit in Zeiten von Covid-19}

https://doi.org/10.1515/bfp-2020-2023

Zusammenfassung: Die Corona-Krise hat weitreichende Konsequenzen für den Betrieb kommunaler Einrichtungen in ganz Europa. Besonders die freiwilligen Einrichtungen der kulturellen Bildung wie Öffentliche Bibliotheken, die jenseits der formalen Bildung einen Beitrag zur Bildungsgerechtigkeit leisten, wurden geschlossen. Öffentliche Bibliotheken mussten ihre Dienstleistungen und Angebote anpassen und neue Formate entwickeln. Der Beitrag zeigt die aktuellen Entwicklungen in Deutschland und Europa auf und beleuchtet die Folgen für die Bildungsgerechtigkeit in Städten und Kommunen.

Schlüsselwörter: Öffentliche Bibliotheken; Covid-19; Bildungsgerechtigkeit; dritte Orte

\section{Public Libraries as Third Places and Educational Justice in the Face of Covid-19}

\begin{abstract}
The Corona crisis has far-reaching consequences for the provision of local public services throughout Europe. This particularly applies to voluntary services within the cultural sector, which contribute to educational justice beyond formal education. Despite the easing up of lockup measures from May 2020, public libraries remained closed and had to adapt their services and develop new formats. The article analyses the current developments in Germany and Europe and highlights the consequences for educational justice at the local level.
\end{abstract}

Keywords: Public libraries; Covid-19; educational justice; third places

\section{Einleitung}

Als sich das Coronavirus im März 2020 in Deutschland ausbreitete, wurde schnell deutlich, dass dies weitreichende Konsequenzen für den Betrieb öffentlicher Einrichtun-

*Kontaktperson: Katja Thiele, kthiele@uni-bonn.de

Prof. Dr. Britta Klagge, klagge@uni-bonn.de gen haben würde. Neben Kindertageseinrichtungen und Schulen gehörten Öffentliche Bibliotheken in vielen deutschen Bundesländern zu den ersten Einrichtungen, die geschlossen wurden. Durch die gravierenden Einschränkungen des öffentlichen Lebens sind sie plötzlich zu „menschenleere[n] Räume[n], [zu] unheimliche[n] Ikonen der Pandemie "1 geworden. Trotz sukzessiver Lockerungsmaßnahmen ab Mai 2020 blieben die meisten Bibliotheken vorerst geschlossen. Ähnliche Entwicklungen können auch in anderen Ländern Europas beobachtet werden. Dies liegt einerseits daran, dass die Kommunen die Maßnahmen zur Lockerung nur nach und nach umsetzen können. Andererseits - und das ist vielleicht viel alarmierender wurde durch die jüngsten Entwicklungen die im Zuge von Digitalisierung und kommunaler Finanzknappheit bzw. Austerität seit vielen Jahren geführte Debatte über die Notwendigkeit des physischen Raums von Bibliotheken wieder entfacht.

Öffentliche Bibliotheken sind dritte Orte der non-formalen Bildung. ${ }^{2}$ Jenseits von Zuhause (erster Ort) und Schule oder Arbeitsplatz (zweiter Ort) tragen sie zu Begegnung und Austausch von Menschen bei. Damit stellen sie eine zentrale Unterstützungsstruktur für Bildungsgerechtigkeit und gesellschaftliche Teilhabe dar. Die Schließung Öffentlicher Bibliotheken ist besonders für diejenigen in unserer Gesellschaft ein Problem, für die die eigenen vier Wände nicht die erforderlichen Bedingungen für die erfolgreiche Teilhabe an Bildungsprozessen bieten. Es ist zwar noch zu früh, um einzuschätzen, welche gesellschaftlichen Auswirkungen die Ausbreitung der Krankheit Covid-19 in der globalen Entwicklung der nächsten Dekade (n) haben wird. In Bezug auf Öffentliche Bibliotheken lässt sich jedoch bereits jetzt erkennen, dass Covid-19 die Entwicklungen der letzten Jahre beschleunigt und negative Auswirkungen auf die gesellschaftliche Teilhabe in Öffentlichen Bibliotheken hat.

Vor dem Hintergrund der Frage um Bildungsgerechtigkeit stellt der Beitrag die Relevanz Öffentlicher Bi-

1 Schuster (2020).

2 Aabø und Audunson (2012). 
bliotheken als dritte Orte heraus und beleuchtet im Anschluss an Überlegungen in den Bibliotheks- und Informationswissenschaften sowie der Stadt-, Wirtschafts- und Bildungsgeographie, welchen Einfluss die aktuellen Maßnahmen auf die Bildungsgerechtigkeit in den Kommunen haben.

\section{Entwicklung von Stadtbibliotheken im Kontext der Covid-19-Pandemie}

Anfang März 2020 kam es im Zuge der Maßnahmen gegen die Ausbreitung des Corona-Virus in ganz Europa zur Schließung von Bibliotheksgebäuden für die Öffentlichkeit. ${ }^{3}$ Die europäische Nicht-Regierungs-Organisation NAPLE (National Authorities on Public Libraries in Europe) hat daraufhin am 30. April einen Bericht über die aktuellen Maßnahmen veröffentlicht. ${ }^{4}$ Der Bericht zeigt, dass sich die Entwicklungen in den 20 Mitgliedsländern ähneln und die lokale Politik jeweils vor den gleichen Herausforderungen steht. Die drei wichtigsten Entwicklungen werden im Folgenden zusammengefasst:

(1) Die Schließung eines Großteils der physischen Einrichtungen Öffentlicher Bibliotheken in Europa war eine der ersten Maßnahmen im Zuge der staatlichen Eindämmungsstrategien. ${ }^{5}$ Der Schwerpunkt der Services liegt seither auf dem Ausbau digitaler Angebote (siehe Punkt 2). Die Rückkehr zum Modell der offenen Bibliothek als physischer Ort, der besucht werden kann, stellt sich als große Herausforderung dar. Die Möglichkeiten der Öffnung hängen stark von den örtlichen Gegebenheiten (Raumkapazität, Personalsituation etc.) und den Sicherheitskonzepten

\footnotetext{
3 Die dargestellten Entwicklungen beziehen sich auf die Mitglieds länder der Organisation NAPLE, d.h. die Länder Belgien (Flandern), Kroatien, Tschechien, Dänemark, Estland, Finnland, Frankreich, Deutschland, Griechenland, Irland, Litauen, Norwegen, Niederlande, Polen, Portugal, Schottland, Slowenien, Spanien, Schweden und die Schweiz. Über ähnliche Entwicklungen in Großbritannien berichtet die Organisation CILIP (Chartered Institute of Library and Information Professionals), s. Poole (2020). Zusätzlich zu diesen Daten wurden (im Rahmen des am Geographischen Institut der Universität Bonn angesiedelten DFG-Projektes Öffentliche Bibliotheken im Spannungsfeld von Finanzknappheit und kommunaler Daseinsvorsorge) Interviews mit Personen in Bibliotheksverwaltungen in Deutschland, England und Schweden geführt und ein Webinar zum Thema Corona und Bibliotheken besucht, das von der Kulturpolitischen Gesellschaft und dem Deutschen Bibliotheksverband am 02.06.2020 durchgeführt wurde, https://kupoge.de/corona-und-bibliotheken/.

4 NAPLE (2020).

5 NAPLE (2020) $2 \mathrm{f}$.
}

der Kommunen bzw. der kommunalen Bibliotheksdienste ab. Da in vielen Großstädten die lokale Bibliothekslandschaft aus mehreren Bibliothekseinrichtungen besteht, die ein gemeinsames Netz bilden, gibt es zumindest in den größeren Städten meist wieder einzelne Öffentliche Bibliotheken, die Dienste vor Ort anbieten. Diese Dienste sind jedoch stark reduziert, beispielsweise auf die Abgabe und Ausleihe von analogen Medien wie Büchern, CDs etc. In vielen Kommunen werden neue Dienste für die kontaktlose Rückgabe und Ausleihe von physischen Bibliotheksmaterialien eingerichtet (Abb. 1). ${ }^{6}$ Für Personen, die den RisikoGruppen angehören, gibt es in einigen Kommunen zusätzlich die Möglichkeit, einen Bücherlieferdienst in Anspruch zu nehmen. Diese neuen Infrastrukturen verursachen unvorhergesehene Kosten für Medien und Technik, Personal und digitale Sicherheit, für die zusätzliche Mittel in den kommunalen Haushalten bereitgestellt werden müssen.

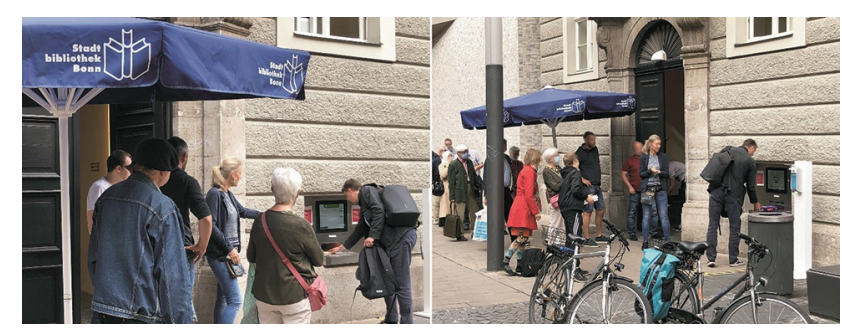

Abb. 1: Eröffnung der neuen Abgabe- und Abholstation der Zentralbibliothek in Bonn im Mai 2020 (Foto: Britta Klagge)

(2) Die Aussetzung des physischen Zugangs zu Öffentlichen Bibliotheken geht zweitens mit einem weiteren Ausbau digitaler Dienste und der verstärkten Nutzung sozialer Medien als Plattform der Öffentlichkeitsarbeit einher. ${ }^{7} \mathrm{Bi}-$ bliotheken haben im Zuge der Digitalisierung immer wieder mit dem Problem der Sichtbarkeit ihrer Angebote zu kämpfen und die Mitarbeiter müssen sich die Frage stellen, wie sie Nutzer erreichen (können). Sie haben in den letzten zwei Jahrzehnten vielerorts mit Erfolg an der Entwicklung neuer Inhalte und Formate gearbeitet, wobei insbesondere Investitionen in ansprechende Architektur und Inneneinrichtung sowie in moderne Technologien, Medienangebote sowie ein weitreichendes und vielfältiges Veranstaltungsund Kursprogramm die Sichtbarkeit Öffentlicher Bibliotheken erhöht haben. Da die physische Ausleihe derzeit nur eingeschränkt möglich ist, wird mehr denn je auf die (Weiter-)Entwicklung von Online-Diensten gesetzt, und es lässt sich seit März 2020 auch eine sprunghafte Steigerung der

6 NAPLE (2020) $5 \mathrm{f}$.
7 NAPLE (2020) $7 \mathrm{ff}$. und 12. 
Akzeptanz bei den Nutzern verzeichnen. Dazu beigetragen haben eine Reihe von veränderten Rahmenbedingungen, beispielsweise die Online-Ausleihe von elektronischen Medien (E-Books, E-Magazine etc.) über eigene Plattformen, ${ }^{8}$ die Lockerung der Beschränkungen für den Zugang $\mathrm{zu}$ digitalen Inhalten (kostenlose Nutzung oder Senkung von Hürden) und die Aufstockung von Lizenzen. Hinzu kommt die Entwicklung eigener Streaming-Dienste (für Filme, Serien oder zum Live-Streamen von Veranstaltungen) und völlig neuer multimedialer Inhalte (z. B. Videotutorials, ${ }^{9}$ Podcasts, ${ }^{10}$ Online-Sprachkurse, digitale StorytellingWorkshops, Gaming-Angebote oder Buchempfehlungen auf YouTube $\left.{ }^{11}\right)$. Im kürzlich erschienenen Sonderheft $B i$ bliotheken des Magazins für Medien, Marketing \& Kommunikation beschreibt Deeg den damit verbundenen Wandel von Bibliotheken hin zu mehr Service- und Kundenorientierung (statt Bestandsorientierung). Bibliotheken werden damit selbst zu Entwicklern von besonderen Angeboten und Events statt nur Medien zu verleihen. ${ }^{12}$ Um Nutzern auch weiterhin In-formationen über Kultur- und Hilfsangebote zugänglich zu machen, werden in Zeiten von Corona außerdem Webseiten, Mailinglisten und soziale Medien genutzt. Auch, wenn Bibliotheken bereits seit mehr als 10 Jahren im Bereich sozialer Medien aktiv sind, werden Plattformen wie Facebook oder Twitter durch die CoronaKrise zum wichtig(st)en Medium im Austausch mit den Nutzern - einerseits, um die Nutzer überhaupt zu erreichen, und andererseits, um die Sichtbarkeit der Bibliothek als öffentliche Einrichtung zu erhöhen. ${ }^{13}$ Interessant ist,

8 In Spanien hat das Angebot virtueller Plattformen für den elektronischen Verleih (bspw. eBiblio, eFilm, Tumblebooks) zu einem raschen Anstieg der Nutzerzahlen geführt, NAPLE (2020) 9.

9 Stellvertretend sei auf ein auf der Video-Plattform YouTube veröffentlichtes Video der Stadtbibliothek Frankfurt am Main verwiesen, in der der Nao-Robotor die neuen Regeln für die Stadtbibliotheken erklärt, https://www.youtube.com/watch?v=m24G8M3qnX4\&t=3s.

10 In den letzten Jahren sind im deutschen Bibliotheksbereich einige Podcasts entstanden: M (Münchner Stadtbibliothek), BibCast (Stadtbibliothek Chemnitz), BücherRausch (Städtische Bibliotheken Dresden).

11 Die britischen Organisationen CILIP und Libraries Connected bieten seit der Corona-Krise den gemeinsamen Dienst National Shelf Service (tägliche E-Book-Empfehlungen von Bibliothekaren) auf ihrem YouTube-Kanal an: https://www.youtube.com/channel/UCPUIql JMOaieXdq-LxKDvWA/live.

12 Deeg (2020).

13 Auf Twitter gibt es derzeit eine ganze Reihe von Hashtags, unter denen die aktuellen Entwicklungen in Deutschland sowohl unter Bibliothekspersonal als auch Nutzern rege diskutiert werden: \#BibchatDE, \#BibliothekenSindDa, \#twittothek, \#LiteraturGesucht, \#BibatHome, \#LibraryTwitter, \#wirbibliotheken. $\mathrm{Zu}$ dem TwitterAccount @BIBChatDE gehört zudem eine eigene Webseite https://www.bibchat.de/, auf der die Diskussionen zum wöchentli- dass Öffentliche Bibliotheken während des Corona-Lockdowns in einigen kleinen Kommunen häufig die einzig verbliebenen und online erreichbaren Kultur- und Bildungseinrichtungen waren bzw. sind. Von den zuständigen Kommunen werden die Online-Auftritte der Öffentlichen Bibliotheken dabei gezielt genutzt, um die Bevölkerung anzusprechen und ihr gesicherte Informationen $\mathrm{zu}$ den Entwicklungen rund um Covid-19 zur Verfügung zu stellen. Von besonderer Relevanz, vor allem im Zusammenhang mit Fake News (u. a. zu Covid-19), ist die Vermittlung von Medienkompetenz durch Öffentliche Bibliotheken - eine Aufgabe, der sich die Bibliotheken allerdings in sehr unterschiedlicher Intensität widmen.

(3) Im Zuge der Corona-Entwicklungen verändert sich drittens die Arbeitswelt der Bibliotheksbeschäftigten ganz erheblich. Dies zeigt sich auch im Bibliotheksalltag. ${ }^{14}$ Der Großteil des Personals in Öffentlichen Bibliotheken muss plötzlich von zu Hause arbeiten und von dort innovative Ideen entwickeln. Wo Arbeit vor Ort möglich ist, werden unter Berücksichtigung der jeweils geltenden CoronaSchutzverordnungen Pläne entwickelt, um einen Teil der Mitarbeiter wieder in den Bibliotheken arbeiten zu lassen und im Rahmen einer schrittweisen Öffnung Angebote vor Ort anzubieten (Abb. 2), beispielsweise im Schichtbetrieb. ${ }^{15}$ Während es in Europa bisher nur in wenigen Fällen zum Abbau von Personal kam (etwa in Finnland durch Nichtverlängerung von zeitlich befristeten Verträgen), wird aus einigen Ländern (u. a. SE, NO, FR, IE, GB, DE) vom Abzug der Bibliotheksmitarbeiter von der Bibliotheksarbeit hin zu anderen Covid-19-bezogenen Aktivitäten berichtet. ${ }^{16}$ In England und Deutschland werden diese Mitarbeiter beispielsweise als Unterstützung der Gesundheitsbehörden bei der Isolation und Versorgung gefährdeter Personen eingesetzt oder übernehmen Telefondienste der kommunalen Verwaltungen. ${ }^{17}$ Vor diesem Hintergrund ist in Teilen der Belegschaft die Sorge vorhanden, dass in Folge der Corona-Krise ein Teil des Personals auf diesem Wege verloren gehen könnte. ${ }^{18}$ Diese Sorge ist insofern berechtigt, als dass diese Maßnahmen mit Entwicklungen korrespon-

chen interaktiven Chat archiviert und gebündelt werden, um den Bibliotheksalltag und die Debatten in Deutschland abzubilden.

14 NAPLE (2020) $4 \mathrm{f}$.

15 In Herten testet die Stadtbibliothek seit April 2020 das Modell der Bibliothek to go. Vorher per Telefon oder E-Mail bestellte Medien können dort in einem extra Bereich der Bibliothek kontaktlos abgeholt werden. Vgl. Beitrag auf dem YouTube-Kanal von cityInfo.TV https://www.youtube.com/watch?v=EDgXIv3_BbE.

16 NAPLE (2020) 5, Poole (2020), eigene Interviews zwischen April und Juli 2020.

17 Poole (2020), eigenes Interview im Mai 2020.

18 NAPLE (2020) 4, Vgl. Anm. 3 zu Webinar. 
dieren, die im Kontext der kommunalen Finanzknappheit und dem verstärkten Bedürfnis nach Kosteneffizienz bereits seit längerem zu beobachten sind. ${ }^{19} \mathrm{Um}$ ihre Kosten zu senken, sind Bibliotheken darüber hinaus zunehmend auf eine Kooperation untereinander sowie mit weiteren Einrichtungen in den Bereichen Bildung und Gesundheit angewiesen. ${ }^{20}$ Dabei setzen sie neben dem Teilen von Ressourcen, Diensten und Aufgaben auch vermehrt auf das Teilen von Personal. ${ }^{21}$ Hinsichtlich der Folgen der CoronaKrise bleibt abzuwarten, inwiefern diese Entwicklungen zu einem Personalabbau führen.

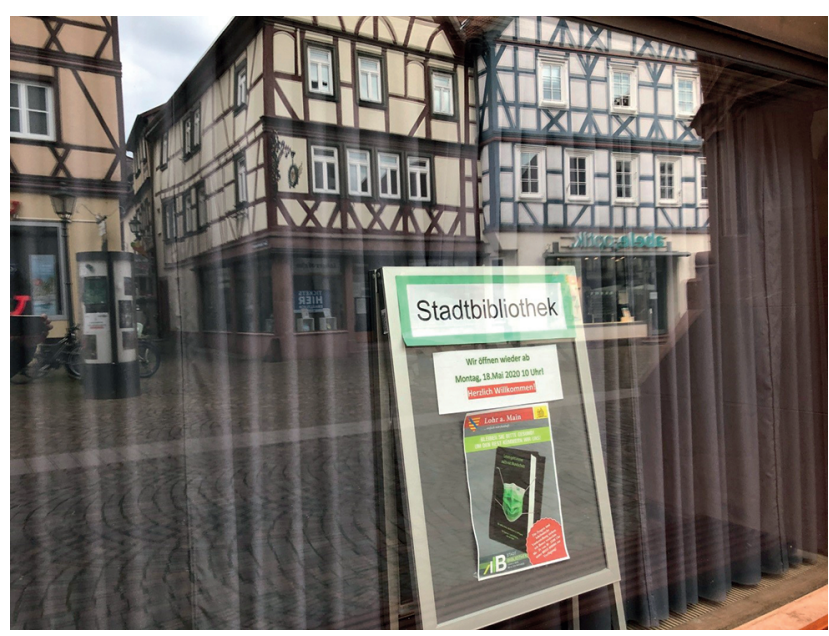

Abb. 2: Stadtbibliothek in Lohr am Main im Juni 2020 (Foto: Britta Klagge)

\section{3 Öffentliche Bibliotheken als dritte Orte}

Angesichts der beschriebenen Herausforderungen im Spannungsfeld von Digitalisierung und kommunaler Austerität haben Öffentliche Bibliotheken in den letzten Jahrzehnten verstärkt daran gearbeitet, ihre Angebote sichtbarer zu machen sowie ihr Image und ihre Rolle als dritte Orte zu stärken..$^{22}$ Der Begriff des dritten Ortes geht zurück auf die in den Sozial- und Kulturwissenschaften diskutierten Konzepte von third space und thirdspace von Bhabha ${ }^{23}$ und Soja. ${ }^{24}$ Gemeint ist ein Raum, der durch seine Offenheit die Begegnung von Menschen unabhängig

19 Düren et al. (2017) $477 \mathrm{f}$.

20 Knoche (2018) 79f., $90 \mathrm{f}$.

21 Thiele (2020) 112.

22 Aabø und Audunson (2012), Hvenegaard Rasmussen (2016).

23 Bhabha (1994).

24 Soja ([1996] 2007). von ihren privaten oder beruflichen Kontexten sowie den damit verbundenen Rollen und Privilegien ermöglicht. Eine für die Stadtgeographie hilfreiche Weiterentwicklung des Begriffes von thirdspace im Sinne von place (konkreter Ort) bietet der Stadtsoziologe Oldenburg. Er versteht thirdplace als einen Ort der Öffentlichkeit außerhalb des eigenen Zuhauses (firstplace) sowie des Ortes, an dem Lohnarbeit oder Schule bzw. Ausbildung stattfindet (secondplace). Nur an einem solchen Ort, der niedrigschwellig für alle Menschen zugänglich ist und Kommunikation und kollaboratives Arbeiten ermöglicht, sei eine neutrale Begegnung zwischen Fremden möglich. ${ }^{25}$ Nur dort könne auch das Gefühl von gesellschaftlicher Teilhabe und Veränderung entstehen. ${ }^{26}$

Auch Öffentliche Bibliotheken können in Anlehnung an Aabø und Audunson und Elmborg als dritte Orte der Begegnung verstanden werden - sowohl für die Begegnung mit Literatur, Technik und ,neuen' Medien als auch für die Begegnung mit Menschen. ${ }^{27}$ Sie sind gerade nicht nur Ausleihstation, sondern soziale Treffpunkte und Kommunikationsorte im Stadtteil - und damit Orte zum ,InGesellschaft-sein' und auch zur Partizipation am öffentlichen Leben. Kommunen bieten ihren Bewohnern durch Öffentliche Bibliotheken zudem einen öffentlich zugänglichen Raum, der nicht-kommerziell ist und niedrigschwellige Bildungs- und Kulturangebote schafft. In vielen Städten hat sich die Bibliothek im Zuge der Digitalisierung außerdem zu einem Ort entwickelt, der die Vernetzung unter den Nutzern stärkt und gleichzeitig eine Kultur des Mit- und Selber-Machens im Sinne der DIY-/DIT-Bewegung(en) fördert. ${ }^{28}$

Durch ihren öffentlichen Auftrag richten sich Öffentliche Bibliotheken mit diesen Angeboten an die Gesamtheit der Bevölkerung unabhängig von ihrem Einkommen und ihrer Herkunft, und damit auch an einkommensschwache bzw. vulnerable Bevölkerungsgruppen. Dazu gehören beispielsweise Geflüchtete oder Obdachlose, die hier Zugang zum lokalen Bildungssystem finden können, sowie Alleinerziehende und ihre Kinder, die in der Corona-Krise besonders belastet sind. Öffentliche Bibliotheken ergänzen so Einrichtungen der Kinder-, Jugend- und Erwachsenenbildung ${ }^{29}$ und dienen der Bildungsgerechtigkeit und Herstellung von gleichwertigen Lebensverhältnissen. ${ }^{30}$ Im Hinblick auf gesellschaftliche Teilhabe können

25 Oldenburg (1989) $20 \mathrm{ff}$.

26 Elmborg (2011) $342 \mathrm{ff}$.

27 Aabø und Audunson (2012) 141, Elmborg (2011) $345 \mathrm{f}$.

28 Hvenegaard Rasmussen (2016), Kurzeja et al. (2020) $163 \mathrm{ff}$.

29 Schüller-Zwierlein und Stang (2010) $521 \mathrm{f}$.

30 Birdi et al. (2008) $580 \mathrm{f}$. 
Öffentliche Bibliotheken als Orte mit einer Art Start-Infrastruktur betrachtet werden, an denen Menschen Hilfe suchen (können). Die Leistungen, die Bibliotheken ihren Nutzern bieten, sind vielfältig und reichen vom Druckservice bis hin zu Hilfestellungen bei der Suche nach Jobs, Kontaktstellen in der Stadtverwaltung oder bei der Recherche zu einer Hausarbeit für die Schule oder Universität. Im Zuge der Übertragung immer neuer Aufgaben an Bibliotheken seit den 1970er Jahren und im Kontext von Digitalisierung oder dem Zuzug von Geflüchteten - zum Beispiel Veranstaltungsorganisation, soziale Bibliotheksarbeit und Sprachkurse für Geflüchtete - sind sie außerdem Anlaufstelle für Menschen mit Problemen aller Art und nichtkommerzieller Aufenthaltsort. „, [F]or connecting vulnerable people to mainstream society“"31 spielen Öffentliche Bibliotheken bzw. ihre physische Präsenz und Zugänglichkeit deshalb besonders in benachteiligten Stadtquartieren und diversen Nachbarschaften eine wichtige Rolle.

\section{Bildungsgerechtigkeit und dritte Orte}

Schon nach wenigen Wochen des Unterrichtens von Kindern in den eigenen vier Wänden wurde deutlich, dass die Corona-Krise existierende Bildungsungleichheiten verstärkt und der Bildungserfolg mehr denn je von der sozialen Herkunft abhängt. ${ }^{32}$ Der spätestens seit den ersten PISA-Studien Anfang der 2000er Jahre umkämpfte Begriff der Bildungsgerechtigkeit spielt bei der Bewertung der Auswirkungen der Corona-Krise eine zentrale Rolle, denn Bildung bewegt sich „im Spannungsfeld zwischen Verteilungs-, Teilhabe- und Anerkennungsgerechtigkeit" ${ }^{\text {“33 }}$ und gilt als eines der wichtigsten „Vehikel zur Verbesserung der Chancen“"34 auf dem Arbeitsmarkt und für Teilhabe an Gesellschaft. Als Einflüsse auf den Lernerfolg werden die Lernatmosphäre und die gezielte Unterstützung von Lernprozessen an non-formalen Orten der (kulturellen) Bildung besonders betont. ${ }^{35}$

Während Bildung und Kultur in der lokalen Verwaltung häufig getrennt abgebildet sind - Bibliotheken gehören als Kultureinrichtungen nicht zur formalen Bildung und dem entsprechenden Ressort - lassen sich die Bereiche in der Praxis kaum voneinander trennen. Kulturein-

31 Birdi et al. (2008) 579.

32 Hurrelmann und Dohmen (2020), Huebener und Schmitz (2020).

33 Stojanov (2011) 27.

34 Kraus (2008) 8.

35 Huebener und Schmitz (2020) 5. richtungen eröffnen Zugänge zu Bildungsprozessen und andersherum. Auf der lokalen Ebene bieten non-formale Bildungsorte wie Öffentliche Bibliotheken zentrale Unterstützungsstrukturen besonders für diejenigen, denen die formalen Lernorte nicht ausreichen. In Anlehnung an die Differenzierung von Bildungsräumen nach ihrer Funktion für das lebenslange Lernen ${ }^{36}$ sind Orte der Öffentlichkeit sogar entscheidend für die Herstellung von Chancengleichheit, weil nur dort Begegnungen außerhalb der eigenen familiären Herkunftskontexte stattfinden (Tab. 1). ${ }^{37}$

Tab. 1: Vier-Säulen Modell des Lebenslangen Lernens

\begin{tabular}{|c|c|c|c|}
\hline \multirow{2}{*}{$\begin{array}{l}\text { Säule } \\
\text { Learning } \\
\text { to be }\end{array}$} & \multicolumn{2}{|c|}{ Bildungsräume und -orte } & \multirow{2}{*}{$\begin{array}{l}\begin{array}{l}\text { Hauptaufgabe / } \\
\text { primäres Ziel }\end{array} \\
\text { Selbstwirksamkeit, } \\
\text { Solidarität, soziale } \\
\text { Kompetenzen, } \\
\text { Ästhetik }\end{array}$} \\
\hline & non-formal & $\begin{array}{l}\text { Privatsphäre } \\
\text { (firstplace) }\end{array}$ & \\
\hline $\begin{array}{l}\text { Learning } \\
\text { to know }\end{array}$ & formal & $\begin{array}{l}\text { Schule } \\
\text { (secondplace) }\end{array}$ & formales Lernen \\
\hline $\begin{array}{l}\text { Learning } \\
\text { to do }\end{array}$ & non-formal & $\begin{array}{l}\text { Arbeitsplatz } \\
\text { (secondplace) }\end{array}$ & $\begin{array}{l}\text { Erwerb von höheren } \\
\text { Qualifikationen und } \\
\text { Kompetenzen }\end{array}$ \\
\hline $\begin{array}{l}\text { Learning to } \\
\text { live together }\end{array}$ & non-formal & $\begin{array}{l}\text { Orte der } \\
\text { Öffentlichkeit } \\
\text { (thirdplace) }\end{array}$ & $\begin{array}{l}\text { Unterstützung der } \\
\text { Lernkompetenzen, } \\
\text { Verständnis anderer } \\
\text { Personen und } \\
\text { Kontexte, Erlernen } \\
\text { sozialer Kompeten- } \\
\text { zen, Erfahrung } \\
\text { gesellschaftlicher } \\
\text { Teilhabe und } \\
\text { Demokratie }\end{array}$ \\
\hline
\end{tabular}

Eigene Zusammenstellung in Anlehnung an den Begriff des dritten Ortes basierend auf Eckert und Tippelt

Trotz aller Debatten über die Möglichkeiten des Ausbaus digitaler Angebote von Bibliotheken sind auch analoge dritte Orte wichtig, an denen man sich begegnen, treffen und austauschen kann. ${ }^{38}$ Öffentliche Bibliotheken vermitteln Lese-, Lern-, Recherche- und Medienkompetenzen, das Verständnis anderer Personen, Erfahrungen und Kontexte und ermöglichen das Erlernen sozialer Kompetenzen. Sie leisten damit einen Beitrag für konkrete Bildungsprozesse und für die Erfahrung von gesellschaftlicher Teilhabe und Demokratie. Die Bedeutung dieser sozialen Funk-

36 Nugel (2016) $16 \mathrm{ff}$.

37 Eckert und Tippelt (2017) 51.

38 U.a. Freytag und Jahnke (2015). 
tionen Öffentlicher Bibliotheken hat im Zusammenhang mit den Fragen der digitalen Teilhabe in den letzten Jahren sogar noch zugenommen. Die Diskussion um ein Recht auf digitale Teilhabe als Daseinsvorsorge wird deshalb auch auf Bundesebene intensiv geführt. Dabei wird explizit betont, dass das deutsche Grundgesetz eine digitale Dimension enthält, insofern, als dass der Zugang zu digitalen Infrastrukturen und die Beteiligung an der Fortentwicklung von Informationstechnik heute zentrale Elemente der Teilhabe an Gesellschaft im Allgemeinen sind. ${ }^{39}$

\section{Gefahr des Verlusts dritter Orte}

Öffentliche Bibliotheken sind Einrichtungen der kommunalen Daseinsvorsorge. Trotz der Bedeutung der öffentlichen Daseinsvorsorge für den Abbau von sozialen Ungleichheiten haben entsprechende Investitionen in Deutschland in den letzten 15 Jahren abgenommen. ${ }^{40}$ Dies gilt besonders für die kommunale Ebene, wo ein Großteil der Leistungen der Daseinsvorsorge erbracht wird. ${ }^{41}$ Hier sind seit Anfang der 2000er-Jahre die Nettoinvestitionen sogar negativ geworden, und es wurde „eine besonders rigide Form der Sparpolitik betrieben“ ${ }^{\text {“2 }}$, die in der (geographischen) wissenschaftlichen Literatur auch als kommunale oder urbane Austerität beschrieben wird. ${ }^{43}$ Die Umsetzung „immer neuer Haushaltskonsolidierungsprogramme“ beschreibt Heinz als Folge dieser Entwicklung. Unter den Kürzungen der letzten zwei Jahrzehnte leiden vor allem freiwillige Leistungen der Kommunen, d.h. ihre Kultur- und Freizeiteinrichtungen. Diese sind, im Gegensatz zu Orten der formalen Bildung, keine Pflichtaufgabe und stehen besonders unter Druck. ${ }^{44}$

Auch Öffentliche Bibliotheken sind vielerorts von Sparprogrammen betroffen. Als Folge arbeiten sie stärker effizienz- und kostenorientiert und reduzieren insbesondere Präsenzdienste und Öffnungszeiten. ${ }^{45}$ Außerdem werden dezentrale Einrichtungen in der Fläche zugunsten zentralisierter Einrichtungen ausgedünnt oder gar geschlossen, und es wird verstärkt auf ehrenamtliche Unterstützung gesetzt. ${ }^{46}$ Einerseits führt dies zu einer Aktivierung der Nachbarschaft, andererseits wird dadurch öffent-

39 Ringwald et al. (2019) 13.

40 Mattert et al. (2017) 19f., 36.

41 Mattert et al. (2017) 19.

42 Mattert et al. (2017) 35.

43 Peck (2015).

44 Heinz (2018).

45 Düren et al. (2017).

46 Thiele (2020). liche Verantwortung abgegeben. Das an physischen Orten vorhandene Angebot wird bezogen auf die Fläche geringer, hinsichtlich der zeitlichen Verfügbarkeit sporadischer sowie insgesamt weniger systematisch und zuverlässig. Die permanente Versorgung im Sinne einer auf den Ausgleich von Bildungsungleichheiten ausgerichteten öffentlichen Daseinsvorsorge war dadurch bereits vor der durch die Covid-19-Pandemie ausgelösten Veränderungen in Gefahr. Die Corona-Krise hat die Herausforderungen für Öffentliche Bibliotheken der letzten Jahre nun an die Oberfläche gespült und droht die Situation der Bildungsgerechtigkeit in den Kommunen in den folgenden drei Punkten zu verschärfen:

- Die Umstellung auf Online-Angebote und die Entwicklung eigener Angebote erfordert finanzielle Mittel für den Ausbau der digitalen Infrastruktur. Hierbei stehen Bibliotheken in Konkurrenz zu Einrichtungen der formalen Bildung (v.a. Schulen), die die gleichen Herausforderung haben.

- Da sich die soziale Interaktion zwischen Menschen nur sehr bedingt ins Digitale verlagern lässt, ist durch die Beschränkung auf digitale Angebote ein Großteil des sozialen Angebots (persönliche Hilfe, Lesenachmittage und andere Veranstaltungen etc.) in den Stadtteilen nicht umsetzbar. Die Kommunen gehen in der Fläche stattdessen zurück zum Modell der Thekenbücherei und die Bedeutung des dritten Ortes und seine sozialen Funktionen, die über Jahre hinweg von Verantwortlichen in der Kulturpolitik und -verwaltung sowie in den Bibliotheken betont und gefördert wurden, rücken dadurch in den Hintergrund.

- Wenn jedoch der physische dritte Ort wegfällt, werden die Verantwortung für die Bildung weiter ins Private verlagert und soziale und regionale Disparitäten verschärft. Die Vermittlung von Lese-, Rechercheund Medienkompetenzen sowie sozialer Kompetenzen kann von den meisten Eltern nicht übernommen werden; insbesondere funktioniert sie unter ungleichen Bedingungen (bzgl. technischer Ausstattung, familiärer Ressourcen) nicht für alle Schüler gleich gut. Auch Erwachsene sind von den Maßnahmen betroffen. Durch kreative Formate und kostenlose Angebote werden zwar neue Nutzer angesprochen, es ist aber nicht bekannt und fraglich, inwiefern ,digitale Randgruppen' erreicht werden (können). Begünstigt werden dadurch einkommensstarke Gruppen, digital affine Menschen und jüngere Altersgruppen.

Die Diskussion über mögliche negative Folgen der Eindämmungsmaßnahmen während der Corona-Krise auf Öffentliche Bibliotheken wird zusätzlich durch die Debatte 
über die finanziellen Folgen der durch die Corona-Krise ausgelösten Rezession befeuert. Die Steuerschätzung des Bundesfinanzministeriums von Mai 2020 geht davon aus, dass die Steuereinnahmen im Jahr 2020 um knapp 100 Milliarden Euro niedriger ausfallen als 2019.47 Zudem werden sowohl auf Ebene des Bundes als auch auf Ebene der Länder und Kommunen hohe Schulden aufgenommen, um mit dem geliehenen Geld die Corona-Folgen zu kompensieren. Das bedeutet, dass in den Kommunen in den kommenden Jahren die finanziellen Spielräume noch geringer werden. Es kann davon ausgegangen werden, dass dies wiederum negative Auswirkungen auf die Investitionen in die öffentliche Infrastruktur hat und eine neue Sparphase vor allem die freiwilligen Leistungen wie Öffentliche Bibliotheken treffen wird.

\section{Fazit}

Trotz aller digitalen Angebote sind besonders die sozialen Funktionen von Öffentlichen Bibliotheken und die damit verbundenen physischen Orte von großer Bedeutung für gesellschaftliche Teilhabe und Bildungsgerechtigkeit in unseren Städten und Kommunen. Dies gilt besonders für diejenigen Teile der Bevölkerung, die in ihrem Alltag nicht über eine entsprechende private digitale und soziale Infrastruktur verfügen. Vor dem Hintergrund der Corona-bedingten Schließung von Bibliotheken nimmt die bereits zwei Jahrzehnte andauernde Debatte um die Notwendigkeit des physischen Raums erneut Fahrt auf. Im Spannungsfeld von Digitalisierung und kommunaler Finanzknappheit beschleunigt die Krise seit Längerem zu beobachtende Entwicklungen. Der in den letzten Jahren viel gepriesene dritte Ort scheint angesichts der CoronaKrise in den Hintergrund zu rücken und die Bibliothek als physischer Ort wird wieder auf das Verleihen von Medien reduziert. Während Öffentliche Bibliotheken ihren Bildungsauftrag in Teilen auch durch digitale Services auffangen können, kann der physische Ort der Begegnung nur sehr bedingt ersetzt werden. Durch den Fokus auf Online-Angebote droht die Gewährleistung ihrer sozialen Funktionen gefährdet zu werden. Diese Gefahr steigt vor allem deshalb, weil Bibliotheken zu den freiwilligen Leistungen der Kommunen gehören und im Zuge der kommunalen Finanzknappheit bereits seit Jahren Einsparpotenziale identifiziert und reduzierte Modelle erprobt wurden. $\mathrm{Zu}$ begegnen ist dieser Gefahr nur durch neue kreative Bibliothekskonzepte, die den physischen Raum

47 Bundesregierung Deutschland (2020).
Bibliothek unter den Bedingungen einer anhaltenden Corona-Pandemie (oder ähnlichen Ereignissen, also mit Abstandsregeln, Desinfektion, Tracking) zugänglich machen und als lebendigen Ort erhalten. Beispiele hierfür gibt es bisher vor allem in den Stadtbibliotheken der Großstädte (Zentralbibliothek in Köln), die über ausreichend große Räumlichkeiten verfügen und Veranstaltungen mit $\mathrm{Ab}$ stand durchführen können.

\section{Literaturverzeichnis}

Aabø, Svanhild; Audunson, Ragnar (2012): Use of library space and the library as place. In: Library \& Information Science Research, 34 (2), 138-49.

Bhabha, Homi K. (1994): The location of culture. London, New York: Routledge.

Birdi, Briony; Wilson, Kerry; Cocker, Joanne (2008): The public library, exclusion and empathy: a literature review. In: Library Review, 57 (8), 576-92.

Bundesregierung Deutschland (2020): Steuerschätzung: Die CoronaKrise ist finanziell zu bewältigen. Verfügbar unter https://www. bundesregierung.de/breg-de/themen/coronavirus/steuerscha etzung-1753004.

Deeg, Christoph (2020): Die Gamified-Corona-Zukunftsbibliothek Sind Bibliotheken der digitalen Herausforderung gewachsen? In: Magazin für Medien, Marketing \& Kommunikation. Sonderheft Bibliotheken - Digital Publishing Report, 50-54. Verfügbar unter https://digital-publishing-report.de/wp-content/uploads/dpr/ ausgaben/dpr_SH_Bibliotheken_2020.pdf\#dpr_SH_Bibliotheke n_2020.indd\%3A.29371\%3A157.

Düren, P.; Landøy, A.; Saarti, J. (2017): New public management and libraries: a success story or just an excuse for cost reduction. In: Library Management, 38 (8/9), 477-87.

Eckert, Thomas; Tippelt, Rudolf (2017): Learning Regions - Learning Cities - Learning Communities: Auf dem Weg zur Gestaltung regionaler Bildungsräume? In: Bildungsgerechtigkeit, hg. v. Thomas Eckert und Burkhard Gniewosz, 49-64. Wiesbaden: Springer Fachmedien GmbH.

Elmborg, James K. (2011): Libraries As the Spaces Between Us: Recognizing and Valuing the Third Space. In: Reference \& User Services Quarterly, 50 (4), 338-50.

Freytag, Tim; Jahnke, Holger (2015): Perspektiven für eine konzeptionelle Orientierung der Bildungsgeographie. In: Geographica Helvetica, 70, 75-88.

Heinz, Werner (2018): Kommunen unter Druck. Transformation kommunaler Handlungs- und Gestaltungsspielräume im Kontext der neoliberalen Globalisierung. Publikation der Bundeszentrale für politische Bildung. Bonn. Verfügbar unter https://www.bpb. de/politik/innenpolitik/stadt-und-gesellschaft/216893/trans formation-kommunaler-handlungsspielraeume.

Huebener, Mathias; Schmitz, Laura (2020): Corona-Schulschließungen: Verlieren leistungsschwächere SchülerInnen den Anschluss? In: DIW aktuell, 30, 06.04.2020. Verfügbar unter http s://www.diw.de/de/diw_01.c.758261.de/publikationen/diw_ak tuell/2020_0030/coronaschulschliessungen_verlieren_leistu ngsschwaechere_schuelerinnen_den_anschluss.html. 
Hurrelmann, Klaus; Dohmen, Dieter (2020): Corona-Krise verstärkt Bildungsungleichheit. Bericht auf dem Deutschen Schulportal, Projekt der Robert-Bosch-Stiftung und der Deutschen Schulakademie gGmbh, 15.04.2020. Verfügbar unter https://deutsches-s chulportal.de/stimmen/das-deutsche-schulbarometer-hurrel mann-dohmen-corona-krise-verstaerkt-bildungsungleichheit/.

Hvenegaard Rasmussen, Casper (2016): The participatory public library. The Nordic experience. In: New Library World, 117 (9/10), 546-56.

Knoche, Michael (2018): Die Idee der Bibliothek und ihre Zukunft. Göttingen: Wallstein Verlag.

Kraus, Josef (2008): Bildungsgerechtigkeit. Essay. In: Aus Politik und Zeitgeschichte, 49, 8-13.

Kurzeja, Matti; Thiele, Katja; Klagge, Britta (2020): Makerspaces dritte Orte für eine zukunftsfähige (Postwachstums-)Gesellschaft. In: Postwachstumsgeographien. Raumbezüge diverser und alternativer Ökonomien, hg. v. Bastian Lange, Martina Hülz, Christian Schulz und Benedikt Schmid, 159-76. Bielefeld: transcript-Verlag.

Mattert, Jana; Valentukeviciute, Laura; Waßmuth, Carl (2017): Gemeinwohl als Zukunftsaufgabe: Öffentliche Infrastrukturen zwischen Daseinsvorsorge und Finanzmärkten. In: Schriften zu Wirtschaft und Soziales, Bd. 20, hg. v. der Heinrich-Böll-Stiftung in Zusammenarbeit mit Gemeingut in BürgerInnenhand (GiB) e. V. Verfügbar unter https://www.gemeingut.org/wp-content/ uploads/2017/06/Endf-Gemeinwohl-als-Zukunftsaufgabe_ Web.pdf.

NAPLE (2020): NAPLE (National Authorities on Public Libraries in Europe): Public Libraries in Europe and COVID-19: Findings from NAPLE Members, 30. April 2020. Verfügbar unter https://www.c ulturaydeporte.gob.es/dam/jcr:fce29ea1-1b83-479b-b674-a592 b255f7e4/naple-narrative-report-european-library-services-dur ing-covid19.pdf.

Nugel, Martin (2016): Stichwort: Bildungsräume - Bildung und Raum. In: Zeitschrift für Erziehungswissenschaft, 19, 9-29.

Oldenburg, Ray (1989): The Great Good Place. Cafés, Coffee Shops, Bookstores, Bars, Hair Salons, and other Hangouts at the Heart Community. New York: Paragon House.

Peck, Jamie (2015): Austerity Urbanism. The Neoliberal Crisis of American Cities. Rosa Luxemburg Stiftung. Verfügbar unter http://www.rosalux-nyc.org/wp-content/files_mf/peck_austeri ty_urbanism_eng93.pdf.

Poole, Nick (2020): A new future for Public Libraries. Beitrag auf Medium, 29.04.2020. Verfügbar unter https://medium.com/@n ick.poole/a-new-future-for-public-libraries-ecad45a78918.

Ringwald, Roman; Schneider, Thomas; Cagan, Tom-Philipp (2019): Smart Cities gestalten: Daseinsvorsorge und digitale Teilhabe sichern. Publikation des Bundesinstituts für Bau-, Stadt- und Raumforschung (BBSR). Bonn. Verfügbar unter https://www.bbs r.bund.de/BBSR/DE/veroeffentlichungen/sonderveroeffentli chungen/2019/smart-cities-daseinsvorsorge-digitale-teilhabedl.pdf?_blob=publicationFile \&v=1.

Schüller-Zwierlein, André; Stang, Richard (2010): Bibliotheken als Supportstrukturen für Lebenslanges Lernen. In: Handbuch Erwachsenenbildung/Weiterbildung, hg. v. Rudolf Tippelt und Aiga von Hippel, 515-26. Wiesbaden: VS Verlag für Sozialwissenschaften.

Schuster, Karin (2020): \#wirbibliotheken: Es gibt viel zu tun! Beitrag zur Vernetzungsaktion \#wirbibliotheken von Katrin Schuster (Direktion Münchner Stadtbibliothek) auf dem Blog der
Münchener Stadtbibliothek. Verfügbar unter https://blog.muen chner-stadtbibliothek.de/wirbibliotheken-es-gibt-viel-zu-tun/.

Soja, Edward W. ([1996] 2007): Thirdspace: Journeys to Loss Angeles and other Real-and-imagined Places. Cambridge, Oxford: Blackwell.

Stojanov, Krassimir (2011): Bildungsgerechtigkeit. Rekonstruktionen eines umkämpften Begriffs. Wiesbaden: VS Verlag für Sozialwissenschaften.

Thiele, Katja (2020): Daseinsvorsorge in Gefahr. Öffentliche Bibliotheken zwischen Digitalisierung und Austerität. In: Geographica Helvetica, 75, 107-22.

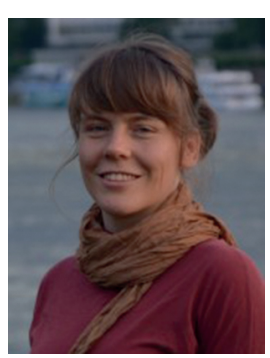

\section{Katja Thiele}

Geographisches Institut

der Universität Bonn

Meckenheimer Allee 166

D-53115 Bonn

kthiele@uni-bonn.de

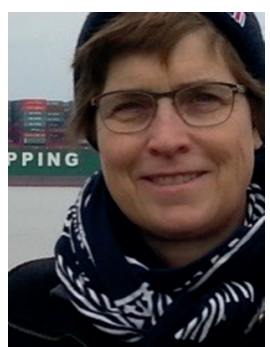

\section{Prof. Dr. Britta Klagge}

Geographisches Institut

der Universität Bonn

Meckenheimer Allee 166

D-53115 Bonn

klagge@uni-bonn.de 